\title{
Direct sums of irreducible operators
}

\author{
by \\ Jun Shen Fang (Tianjin), Chun-Lan Jiang (Shijiazhuang) and \\ Pei Yuan Wu (Hsinchu)
}

\begin{abstract}
It is known that every operator on a (separable) Hilbert space is the direct integral of irreducible operators, but not every one is the direct sum of irreducible ones. We show that an operator can have either finitely or uncountably many reducing subspaces, and the former holds if and only if the operator is the direct sum of finitely many irreducible operators no two of which are unitarily equivalent. We also characterize operators $T$ which are direct sums of irreducible operators in terms of the $C^{*}$-structure of the commutant of the von Neumann algebra generated by $T$.
\end{abstract}

1. Introduction. A bounded linear operator on a complex separable Hilbert space $H$ is irreducible if it has no reducing subspace other than $\{0\}$ and $H$; otherwise, it is reducible. In this paper, we are concerned with the problem of characterizing operators which are expressible as the direct sum of irreducible operators. Examples of such operators include any finite-dimensional operator, compact operator, completely nonnormal essentially normal operator, completely nonnormal hyponormal operator with finite multiplicity (cf. [7, Section 2.1]) and any Cowen-Douglas operator (cf. [3, Proposition 1.18]). On the other hand, not every operator can be expressed as such a direct sum. This is the case even for normal operators since it can be easily seen that a normal operator is irreducible if and only if it acts on a one-dimensional space, and thus it is the direct sum of irreducible operators if and only if it is diagonalizable. In particular, the bilateral shift (the operator of multiplication by the independent variable on the $L^{2}$-space of the unit circle) cannot be the direct sum of irreducible operators.

In Section 2 below, we first show in Theorem 2.1 that no operator can have countably infinitely many reducing subspaces, that is, the number of reducing subspaces of any operator is either finite or $\aleph_{1}$, the cardinal number of the real numbers. Moreover, an operator has finitely many reducing

2000 Mathematics Subject Classification: 47A15, 47C15.

Key words and phrases: irreducible operator, reducing subspace, von Neumann algebra. 
subspaces if and only if it is the direct sum of finitely many irreducible operators no two of which are unitarily equivalent. These are proved by making use of the structure theorem of two projections (Lemma 2.2).

An equivalent condition for irreducibility can be formulated in terms of the von Neumann algebra generated by the operator. Indeed, if $W^{*}(T)$ denotes the von Neumann algebra generated by an operator $T$ on $H$ and $W^{*}(T)^{\prime}$ denotes its commutant, then using the von Neumann double commutant theorem we can easily show the equivalence of the following three conditions:

(1) $T$ is irreducible,

(2) $\operatorname{dim} W^{*}(T)^{\prime}=1$, and

(3) $W^{*}(T)$ equals $\mathcal{B}(H)$, the algebra of all operators on $H$.

In Section 3, we will generalize this to direct sums of irreducible operators. We show in Theorem 3.1 that $T$ is such a direct sum if and only if $W^{*}(T)^{\prime}$ is *-isomorphic to the direct sum of full matrix algebras $M_{n_{i}}(\mathbb{C})$ with various sizes $n_{i}, 1 \leq n_{i} \leq \infty$. Here $M_{n_{i}}(\mathbb{C}), 1 \leq n_{i} \leq \infty$, denotes the algebra of all $n_{i}$-by- $n_{i}$ complex matrices, and $M_{\infty}(\mathbb{C})$ is understood to be $\mathcal{B}\left(l^{2}\right)$. As a corollary (Corollary 3.2), we have the equivalence of $T$ being the direct sum of finitely many irreducible operators and $\operatorname{dim} W^{*}(T)^{\prime}<\infty$.

If all the $n_{i}$ 's are finite in the above representation for $W^{*}(T)^{\prime}$, that is, if $W^{*}(T)^{\prime}$ is $*$-isomorphic to the direct sum of full finite matrix algebras, then $W^{*}(T)^{\prime}$, as an approximately finite algebra, can be characterized in terms of its (scaled ordered) $K_{0}$-group. (For results on the $K$-theory of $C^{*}$-algebras, the reader can consult [13].) However, in our present situation, the full infinite matrix algebra $M_{\infty}(\mathbb{C})$ may appear, which renders the $K_{0}$-group characterization inappropriate. In our final section, we show that for this case the characterization can be obtained in terms of the semigroup $V\left(W^{*}(T)^{\prime}\right)$.

We conclude this section with two further remarks. Firstly, it is known that on an infinite-dimensional separable Hilbert space $H$, there are plenty of irreducible operators in the sense that such operators are dense in $\mathcal{B}(H)$ in the norm topology (cf. [4]). In [4], it was asked whether reducible operators are also dense. This is answered affirmatively by Voiculescu [12]. In fact, an even stronger result is true, namely, for any operator $T$ and any $\varepsilon>0$, there is a compact operator $K$ with $\|K\|<\varepsilon$ such that $T+K$ is the direct sum of infinitely many irreducible operators (cf. also [6, Proposition 4.21(iv), (v)]).

Secondly, although not every operator is the direct sum of irreducible operators, every one can be expressed as the direct integral of irreducible ones. This is what the next proposition says.

Proposition 1.1. Every operator is the direct integral of irreducible operators. 
Proof. This is an easy consequence of [1, Theorem 3.6] on the direct integral decomposition of operator algebras. Indeed, since for any operator $T$, the weakly closed algebra $\operatorname{Alg} T$ generated by $T$ and $I$ can be expressed as $\int_{\Lambda}^{\oplus} \mathcal{A}_{\lambda} d \mu(\lambda)$, where $\Lambda$ is a separable metric space, $\mu$ is (the completion of) a $\sigma$-finite regular Borel measure on $\Lambda$, and $\mathcal{A}_{\lambda}$ is a weakly closed irreducible operator algebra for almost all $\lambda$ in $\Lambda$ (an operator algebra is irreducible if it has no nontrivial reducing subspace), we have $T=\int_{\Lambda}^{\oplus} T_{\lambda} d \mu(\lambda)$, where $T_{\lambda}$ is in $\mathcal{A}_{\lambda}$ for almost all $\lambda$. Hence $\operatorname{Alg} T \subseteq \int_{\Lambda}^{\oplus} \operatorname{Alg} T_{\lambda} d \mu(\lambda) \subseteq \int_{\Lambda}^{\oplus} \mathcal{A}_{\lambda} d \mu(\lambda)=$ $\operatorname{Alg} T$, which implies that $\operatorname{Alg} T_{\lambda}=\mathcal{A}_{\lambda}$ for almost all $\lambda$. The irreducibility of $\mathcal{A}_{\lambda}$ then implies that of $T_{\lambda}$. Thus $T=\int_{\Lambda}^{\oplus} T_{\lambda} d \mu(\lambda)$ is the asserted decomposition of $T$.

For any $C^{*}$-algebra $\mathcal{A}$ and natural number $n$, let $M_{n}(\mathcal{A})$ denote the $C^{*}$-algebra of $n$-by- $n$ matrices with entries from $\mathcal{A}$.

2. Number of reducing subspaces. The main result of this section is the following theorem.

THEOREM 2.1. The number of reducing subspaces of any operator is either finite or uncountably infinite. The former case occurs if and only if the operator is the direct sum of finitely many irreducible operators $\sum_{i=1}^{n} \oplus T_{i}$ with $T_{i}$ and $T_{j}$ unitarily inequivalent for any $i \neq j$. In this case, the number of reducing subspaces is $2^{n}$.

The preceding result has an analogue in a different context: the number of invariant subspaces of any operator on a finite-dimensional space is either finite or uncountably infinite, the former case occurring if and only if the operator is cyclic (cf. [9]).

To prove Theorem 2.1, we need three lemmas. The first one is a structure theorem for two arbitrary (orthogonal) projections. This result has appeared repeatedly in the literature before; the version we adopt below is from [5].

Lemma 2.2. Let $P$ and $Q$ be two arbitrary projections on a Hilbert space. Then there is a unitary operator $U$ such that

$$
U^{*} P U=\left(\begin{array}{cc}
I_{1} & 0 \\
0 & 0
\end{array}\right) \oplus I_{2} \oplus I_{3} \oplus 0 \oplus 0
$$

and

$$
U^{*} Q U=\left(\begin{array}{cc}
A & B \\
B & I_{1}-A
\end{array}\right) \oplus I_{2} \oplus 0 \oplus I_{4} \oplus 0
$$

on the space $H_{1} \oplus H_{1} \oplus H_{2} \oplus H_{3} \oplus H_{4} \oplus H_{5}$, where $A$ is a positive contraction on $H_{1}$ and $B$ is the positive square root of $A\left(I_{1}-A\right)$. We may require that $0<A \leq \frac{1}{2} I_{1}$, in which case $A$ is unique up to unitary equivalence.

The preceding lemma is used to prove 
LEMMA 2.3. If $T$ has countably many reducing subspaces, then $W^{*}(T)^{\prime}$ is abelian.

Proof. Let $P$ and $Q$ be two projections in $W^{*}(T)^{\prime}$ represented as in Lemma 2.2 with $0<A \leq \frac{1}{2} I_{1}$. Since $P$ and $Q$ both commute with $T$, a simple computation shows that $T$ is of the form $T_{1} \oplus T_{1} \oplus \sum_{i=2}^{5} \oplus T_{i}$ on $H_{1} \oplus H_{1} \oplus \sum_{i=2}^{5} \oplus H_{i}$ with $T_{1} A=A T_{1}$. For each complex scalar $\lambda$, let $M_{\lambda}$ be the subspace $\left\{\lambda B x \oplus x \oplus 0 \oplus 0 \oplus 0 \oplus 0: x \in H_{1}\right\}$. It is easily seen that the $M_{\lambda}$ 's are all reducing subspaces of $T$ and are distinct if $H_{1} \neq\{0\}$. Since $T$ has only countably many reducing subspaces, this forces $H_{1}=\{0\}$. Hence $P=I_{2} \oplus I_{3} \oplus 0 \oplus 0$ and $Q=I_{2} \oplus 0 \oplus I_{4} \oplus 0$ commute. Since the von Neumann algebra $W^{*}(T)^{\prime}$ is generated by the projections it contains, we infer that $W^{*}(T)^{\prime}$ is abelian.

Recall that a projection $p$ in a $C^{*}$-algebra is minimal if there is no projection $q$ other than 0 and $p$ such that $p q=q$.

Lemma 2.4. Let $P$ be a projection in $W^{*}(T)^{\prime}$. Then $T \mid \operatorname{ran} P$ is irreducible if and only if $P$ is a minimal projection in $W^{*}(T)^{\prime}$.

This is an easy consequence of the definitions of irreducibility and minimal projection.

We need one more lemma.

LEMMA 2.5. Let $A$ and $B$ be irreducible operators on $H$ and $K$, respectively. Then $A$ and $B$ are unitarily equivalent if and only if there is a nonzero operator $X$ such that $X A=B X$ and $X A^{*}=B^{*} X$.

Proof. Assume that $X A=B X$ and $X A^{*}=B^{*} X$ for some $X \neq 0$. It is easily seen that ker $X$ and $\overline{\operatorname{ran} X}$ are reducing subspaces of $A$ and $B$, respectively. If $\operatorname{ker} X \neq\{0\}$, then by the irreducibility of $A$ we have $\operatorname{ker} X=H$, i.e. $X=0$, which contradicts our assumption. Hence $\operatorname{ker} X=\{0\}$, i.e. $X$ is one-to-one. In a similar fashion, we infer that $\overline{\operatorname{ran} X}=K$, i.e. $X$ has dense range. Therefore, the polar decomposition of $X$ yields $X=U P$, where $U$ is unitary and $P=\left(X^{*} X\right)^{1 / 2} \geq 0$. Since $X^{*} X A=X^{*} B X=A X^{*} X$, we have $P A=A P$. Hence $U A P=U P A=X A=B X=B U P$. Note that $P$ also has dense range. From the above, we conclude that $U A=B U$, which shows the unitary equivalence of $A$ and $B$ as asserted.

We are now ready for the proof of Theorem 2.1.

Proof of Theorem 2.1. Assume that an operator $T$ has a countably infinite number of reducing subspaces. This implies, by Lemma 2.3, that $W^{*}(T)^{\prime}$ is abelian. Hence it is generated by some Hermitian operator $A$ (cf. [10, Theorem 7.12]). Note that $\sigma(A)$, the spectrum of $A$, cannot be a finite set for otherwise $A$ would be of the form $\sum_{i=1}^{n} \oplus \lambda_{i} I_{i}$ and $W^{*}(A)$ would consist of operators of the form $\sum_{i=1}^{n} \oplus \alpha_{i} I_{i}$ with scalars $\alpha_{i}$, which implies 
that $W^{*}(A)=W^{*}(T)^{\prime}$ contains only finitely many projections, contradicting our assumption. Thus we can decompose $\sigma(A)$ into countably infinitely many mutually disjoint Borel subsets each having a strictly positive spectral measure. The spectral projections corresponding to various unions of such subsets are all in $W^{*}(A)=W^{*}(T)^{\prime}$. Since there are uncountably many of them, this again contradicts our assumption. Thus the number of reducing subspaces of $T$ cannot be countably infinite.

Now assume that $T$ has finitely many reducing subspaces. By Lemma 2.3, $W^{*}(T)^{\prime}$ is abelian. Let $P_{1}, \ldots, P_{n}$ be the minimal projections in it. Since the $P_{j}$ 's are commuting, it is easily seen that they are mutually orthogonal and have sum equal to $I$. Let $T=\sum_{i=1}^{n} \oplus T_{i}$ on $H=\sum_{i=1}^{n} \oplus \operatorname{ran} P_{i}$. Then the $T_{i}$ 's are irreducible by Lemma 2.4 . Next we prove that no two of the $T_{i}$ 's are unitarily equivalent. For this, assume otherwise that there is a unitary operator $U$ such that $U T_{i}=T_{j} U$, where $1 \leq i<j \leq m$. For any scalar $\lambda$, let

$$
M_{\lambda}=\left\{0 \oplus \ldots \oplus \underset{i \mathrm{th}}{x} \oplus 0 \oplus \ldots \oplus 0 \oplus \underset{j \mathrm{th}}{\lambda U_{\mathrm{th}}} \oplus \ldots \oplus 0: x \in H_{i}\right\} .
$$

Then the $M_{\lambda}$ 's are distinct reducing subspaces of $T$. Since there are infinitely many of them, this contradicts our assumption on $T$.

Conversely, assume that $T=\sum_{i=1}^{n} \oplus T_{i}$ on $H=\sum_{i=1}^{n} \oplus H_{i}$, where the $T_{i}$ 's are all irreducible and no two of them are unitarily equivalent. Let $P=$ $\left[P_{i j}\right]_{i, j=1}^{n}$ be a projection commuting with $T$. Then $P_{i j} T_{j}=T_{i} P_{i j}$ for all $i$ and $j$. From this we obtain $P_{i j} T_{j}^{*}=P_{j i}^{*} T_{j}^{*}=\left(T_{j} P_{j i}\right)^{*}=\left(P_{j i} T_{i}\right)^{*}=T_{i}^{*} P_{j i}^{*}=$ $T_{i}^{*} P_{i j}$. Since $T_{i}$ and $T_{j}$ are irreducible and are not unitarily equivalent for $i \neq j$, Lemma 2.5 implies that $P_{i j}=0$ and hence also $P_{j i}=0$. Thus $P_{i i}$ is a projection commuting with $T_{i}$. The irreducibility of $T_{i}$ implies that $P_{i i}=0$ or $I_{i}$. This shows that $P$ is one of the $2^{n}$ projections obtained by taking the direct sum of some of the $I_{i}$ 's with the 0's. Equivalently, this says that the reducing subspaces of $T$ are the $2^{n}$ subspaces obtained by taking the direct sum of some of the $H_{i}$ 's with the $\{0\}$ 's, completing the proof.

3. Full matrix algebras. In this section, we will characterize the direct sum of irreducible operators in terms of the $C^{*}$-algebra structure of the commutant of its generated von Neumann algebra.

For any operator $T$ on $H$ and any integer $n, 1 \leq n \leq \infty$, let $T^{(n)}$ denote the direct sum of $n$ copies of $T$ on $H^{(n)}=H \oplus \ldots \oplus H$ ( $n$ copies).

TheOREM 3.1. An operator $T$ on $H$ is the direct sum of irreducible operators, say, $\sum_{i=1}^{n} \oplus T_{i}^{\left(n_{i}\right)}$ on $\sum_{i=1}^{n} \oplus H_{i}^{\left(n_{i}\right)}$, where $1 \leq n \leq \infty$, $1 \leq n_{i} \leq \infty$ for all $i$ and the $T_{i}$ 's are pairwise unitarily inequivalent, if and only if $W^{*}(T)^{\prime}$ is $*$-isomorphic to $\sum_{i=1}^{n} \oplus M_{n_{i}}(\mathbb{C})$. Moreover, the $T_{i}$ 's are unique up to permutation and unitary equivalence. More precisely, if 
$T=\sum_{k=1}^{m} \oplus S_{k}^{\left(m_{k}\right)}$ is another direct sum of irreducible operators with pairwise unitarily inequivalent $S_{k}$ 's, then $n=m$ and there is a permutation $\pi$ of $\{1, \ldots, n\}$ and a unitary operator $U$ in $W^{*}(T)^{\prime}$ such that $n_{i}=m_{\pi(i)}$ and $U T_{i}=S_{\pi(i)} U$ for all $i$.

Since every finite-dimensional (unital) $C^{*}$-algebra is $*$-isomorphic to the direct sum of finitely many full (finite) matrix algebras (cf. [11, Theorem 11.2]), an easy consequence of the preceding theorem is

COROLlary 3.2. $T$ is the direct sum of finitely many irreducible operators if and only if $\operatorname{dim} W^{*}(T)^{\prime}<\infty$.

We need the following lemma for the proof of Theorem 3.1.

LEMMA 3.3. If $T$ is irreducible on $H$ and $X$ is such that $X T=T X$ and $X T^{*}=T^{*} X$, then $X$ is a scalar multiple of identity.

Proof. Since $X^{*} X$ commutes with $T$, the same is true for any spectral projection $P$ of $X^{*} X$. The irreducibility of $T$ then implies that $P=0$ or $I$. Thus the spectrum of $X^{*} X$ must be a singleton $\{\alpha\}$ and hence $X^{*} X=\alpha I$. On the other hand, from the assumptions $X T=T X$ and $X T^{*}=T^{*} X$ we also deduce that $\operatorname{ker} X$ is a reducing subspace of $T$. Thus $\operatorname{ker} X=\{0\}$ or $H$. This says that either $X$ is one-to-one or $X=0$. Similarly, by considering $\overline{\operatorname{ran} X}$, we deduce that either $X$ has dense range or $X=0$. Thus for our purpose we may assume that $X$ is one-to-one with dense range. Hence $X=$ $U\left(X^{*} X\right)^{1 / 2}=\sqrt{\alpha} U$, where $U$ is unitary, by polar decomposition. We may assume that $\alpha \neq 0$. Then $U T=T U$ and $U T^{*}=T^{*} U$. Arguing as above, we obtain $U=\beta I$. Thus $X=\sqrt{\alpha} \beta I$ is a scalar multiple of identity.

Proof of Theorem 3.1. Assume $T=\sum_{i=1}^{n} \oplus T_{i}^{\left(n_{i}\right)}$ on $H=\sum_{i=1}^{n} \oplus H_{i}^{\left(n_{i}\right)}$, where the $T_{i}$ 's are pairwise unitarily inequivalent irreducible operators. If $X$ is an operator in $W^{*}(T)^{\prime}$, then $X=\sum_{i=1}^{n} \oplus X_{i}$ with $X_{i}$ in $W^{*}\left(T_{i}^{\left(n_{i}\right)}\right)^{\prime}$ by Lemma 2.5. Letting $X_{i}=\left[Y_{j k}^{i}\right]_{j, k=1}^{n_{i}}$, we see that $Y_{j k}^{i}$ belongs to $W^{*}\left(T_{i}\right)^{\prime}$. Therefore $Y_{j k}^{i}$ is a scalar multiple of identity by Lemma 3.3. Say, $Y_{j k}^{i}=\lambda_{j k}^{i} I_{i}$, where $I_{i}$ is the identity operator on $H_{i}$. Then $X=\sum_{i=1}^{n} \oplus\left[\lambda_{j k}^{i} I_{i}\right]_{j, k=1}^{n_{i}}$. Obviously, the mapping $X \mapsto \sum_{i=1}^{n} \oplus\left[\lambda_{j k}^{i}\right]_{j, k=1}^{n_{i}}$ defines a *-isomorphism from $W^{*}(T)^{\prime}$ onto $\sum_{i=1}^{n} \oplus M_{n_{i}}(\mathbb{C})$.

Conversely, let $\Phi$ be a $*$-isomorphism from $W^{*}(T)^{\prime}$ onto

$$
\mathcal{A} \equiv \sum_{i=1}^{n} \oplus M_{n_{i}}(\mathbb{C}),
$$

and let $E_{i j}$ denote the element $0 \oplus \ldots \oplus e_{i j} \oplus \ldots \oplus 0$ in $\mathcal{A}$, where $e_{i j}$ is the $n_{i}$-by- $n_{i}$ matrix whose $(j, j)$-entry equals 1 and all others equal 0 . Then the $\Phi^{-1}\left(E_{i j}\right)$ 's are mutually orthogonal minimal projections in $W^{*}(T)^{\prime}$ with 
sum equal to $I$. Obviously, $\Phi^{-1}\left(E_{i j}\right) H$ is a reducing subspace of $T$ with $T_{i j} \equiv T \mid \Phi^{-1}\left(E_{i j}\right) H$ irreducible (by Lemma 2.4), and $T=\sum_{i j} \oplus T_{i j}$. Since for any pair $j$ and $k$ the matrices $E_{i j}$ and $E_{i k}$ are unitarily equivalent (via a unitary operator, say, $U$ in $\mathcal{A}$ ), we infer that $T_{i j}$ and $T_{i k}$ are unitarily equivalent (via the unitary $\Phi^{-1}(U) \mid \Phi^{-1}\left(E_{i j}\right) H$ ). Thus $T$ is the direct sum of irreducible operators $\sum_{i=1}^{n} \oplus T_{i 1}^{\left(n_{i}\right)}$ as asserted.

To prove the uniqueness, let $T=\sum_{k=1}^{m} \oplus S_{k}^{\left(m_{k}\right)}$ on $H=\sum_{k=1}^{m} \oplus L_{k}^{\left(m_{k}\right)}$ be another direct sum of irreducible operators for $T$ with pairwise unitarily inequivalent $S_{k}$ 's, where $1 \leq m \leq \infty$ and $1 \leq m_{k} \leq \infty$ for all $k$. If $P_{k l}$, $1 \leq k \leq m$ and $1 \leq l \leq m_{k}$, denotes the projection from $H$ onto the $l$ th component of $L_{k}^{\left(m_{k}\right)}$, then the mutually orthogonal projections $F_{k l} \equiv \Phi\left(P_{k l}\right)$ in $\mathcal{A}$ are such that $\sum_{k, l} F_{k l}=I$. Moreover, since each $F_{k l}$ is minimal by Lemma 2.4, it can only "live" in some $M_{n_{i}}(\mathbb{C})$ and can only have rank one. Also note that for any fixed $k$, the different $F_{k l}$ 's are all in the same $M_{n_{i}}(\mathbb{C})$ with $\sum_{l} F_{k l}=I_{n_{i}}$, the identity matrix of size $n_{i}$. This is because for a fixed $k$, the different $P_{k l}$ 's are unitarily equivalent via a unitary operator in $W^{*}(T)^{\prime}$, and thus the different $F_{k l}$ 's are unitarily equivalent via a unitary operator in $\mathcal{A}$. This latter unitary operator, being a direct sum of operators from the $M_{n_{j}}(\mathbb{C})$ 's, can intertwine only operators in the same $M_{n_{i}}(\mathbb{C})$. Since $\sum_{l} F_{k l}=I_{n_{i}}$ and the mutually orthogonal $F_{k l}$ 's each have rank one, we infer that $m_{k}=n_{i}$ and the $F_{k l}$ 's (for different l's) are simultaneously unitarily equivalent to the $E_{i j}$ 's (for different $j$ 's). From $\sum_{k, l} F_{k l}=I=\sum_{i, j} E_{i j}$ and the above, we conclude that $m=n$ and, after a permutation of the indices, the $F_{k l}$ 's (for different $k$ 's and l's) are simultaneously unitarily equivalent to the $E_{i j}$ 's (for different $i$ 's and $j$ 's). Our assertion on the uniqueness of the irreducible summands for $T$ then follows by applying $\Phi^{-1}$ to the $F_{k l}$ 's and the intertwining unitary operator in $\mathcal{A}$.

We next consider the problem when two operators have isomorphic reducing subspace lattices. When the operators are normal, this has been solved by Conway and Gillespie [2]. Using their result, we may settle the problem when the two operators are both direct sums of irreducible ones. This covers in particular the cases of operators on finite-dimensional spaces and compact operators.

For any operator $T$, let Red $T$ denote the lattice of its reducing subspaces.

Proposition 3.4. Let $A=\sum_{j=1}^{n} \oplus A_{j}^{\left(n_{j}\right)}$ and $B=\sum_{k=1}^{m} \oplus B_{k}^{\left(m_{k}\right)}$ be direct sums of irreducible operators with pairwise unitarily inequivalent $A_{j}$ 's and $B_{k}$ 's, where $1 \leq n, m \leq \infty$ and $1 \leq n_{j}, m_{k} \leq \infty$ for all $j$ and $k$. Then Red $A$ is isomorphic to $\operatorname{Red} B$ if and only if $n=m$ and there is a permutation $\pi$ of $\{1, \ldots, n\}$ such that $n_{j}=m_{\pi(j)}$ for all $j$.

To prove this, we need the following 
LEMma 3.5. If $T$ is irreducible, then, for any $1 \leq n \leq \infty$, $\operatorname{Red} T^{(n)}$ is isomorphic to $\operatorname{Red} I_{n}$, where $I_{n}$ denotes the identity operator on an $n$-dimensional space.

Proof. If $P=\left[P_{i}^{j}\right]_{i, j=1}^{n}$ is any projection commuting with $T^{(n)}$, then for any $i$ and $j$ we deduce using Lemma 3.3 that $P_{i j}=\lambda_{i j} I$, where $\lambda_{i j}$ is some scalar. The mapping $P \mapsto\left[\lambda_{i j}\right]_{i, j=1}^{n}$ then induces a lattice isomorphism from $\operatorname{Red} T^{(n)}$ onto Red $I_{n}$.

Proof of Proposition 3.4. Using Lemma 2.5, we may infer that $\operatorname{Red} A$ and $\sum_{j} \oplus \operatorname{Red} A_{j}^{\left(n_{j}\right)}$ are isomorphic. This latter lattice is isomorphic to $\sum_{j} \oplus \operatorname{Red}(1 / j) I_{n_{j}}$ (by Lemma 3.5) or $\operatorname{Red} \sum_{j} \oplus(1 / j) I_{n_{j}}$. Hence $\operatorname{Red} A$ is isomorphic to Red $\sum_{j} \oplus(1 / j) I_{n_{j}}$. A similar assertion holds for $B$. Hence if $\operatorname{Red} A$ and $\operatorname{Red} B$ are isomorphic, then the same is true for $\operatorname{Red} \sum_{j} \oplus(1 / j) I_{n_{j}}$ and $\operatorname{Red} \sum_{k} \oplus(1 / k) I_{m_{k}}$. For normal operators, this implies that $n=m$ and there is a permutation $\pi$ of $\{1, \ldots, n\}$ such that $n_{j}=m_{\pi(j)}$ for all $j$ (cf. [2, Theorem 3.2]). A reversal of the above implications yields the converse.

The next result will be useful in Section 4 .

Proposition 3.6. If $T^{(k)}$ is a direct sum of irreducible operators, where $k$ is a natural number, then so is $T$.

Proof. Assume that $T^{(k)}$ is unitarily equivalent to the direct sum $S \equiv$ $\sum_{i=1}^{n} \oplus T_{i}{ }^{\left(n_{i}\right)}$, where $1 \leq n \leq \infty, 1 \leq n_{i} \leq \infty$ for all $i$ and the $T_{i}$ 's are pairwise unitarily inequivalent irreducible operators. Then there are mutually orthogonal projections $P_{j}, j=1, \ldots, k$, commuting with $S$ and satisfying $\sum_{j} P_{j}=I$ such that $S \mid\left(\operatorname{ran} P_{j}\right), j=1, \ldots, k$, are mutually unitarily equivalent. Using Lemma 2.5, we deduce that $P_{j}$ is of the form $\sum_{i} \oplus Q_{i j}$, where the $Q_{i j}$ 's are mutually orthogonal projections commuting with $T_{i}^{\left(n_{i}\right)}$ and satisfying $\sum_{j} Q_{i j}=I_{i}$ such that $T_{i}^{\left(n_{i}\right)} \mid\left(\operatorname{ran} Q_{i j}\right), j=1, \ldots, k$, are mutually unitarily equivalent. Thus we are reduced to proving the following: if $A^{(k)}$ is unitarily equivalent to $B^{(n)}, 1 \leq n \leq \infty$, where $B$ is irreducible, then $A$ is a direct sum of irreducible operators. We may further assume that $n=\infty$ for otherwise $W^{*}\left(A^{(k)}\right)^{\prime}=M_{k}\left(W^{*}(A)^{\prime}\right)$ is finite-dimensional by Corollary 3.2, which implies the same for $W^{*}(A)^{\prime}$ and thus our assertion for $A$ follows by Corollary 3.2 again. Under the assumption $n=\infty, A^{(k)}$ is unitarily equivalent to $C^{(k)}$, where $C=B^{(\infty)}$. The unitary equivalence of $A$ and $C$ then follows from an argument analogous to the proof of the first test problem in $[8]$.

4. $K$-theoretic characterization. In the preceding section, direct sums of irreducible operators are characterized in terms of the structure 
of certain $C^{*}$-algebras. We now proceed to describe the latter in terms of some ingredients from $K$-theory.

If $\mathcal{A}$ is the $C^{*}$-algebra $\sum_{i=1}^{n} \oplus M_{n_{i}}(\mathbb{C})$ with $1 \leq n \leq \infty$ and $1 \leq n_{i}<\infty$ for all $i$, then $\mathcal{A}$ is an approximately finite algebra and hence can be characterized by its (scaled ordered) $K_{0}$-group (cf. [13, Theorem 12.1.3]). However, if we allow some $n_{i}$ 's to be $\infty$, then the $K_{0}$-group can no longer distinguish two such algebras. This is because the $K_{0}$-group of $M_{\infty}(\mathbb{C})$ is trivial (cf. [13, Examples 6.2.3]). However, for any $C^{*}$-algebra $\mathcal{A}$ its $K_{0}$-group is defined through an abelian semigroup $V(\mathcal{A})$, and it turns out that the latter is strong enough to distinguish $M_{n}(\mathbb{C})$ for finite and infinite values of $n$. Indeed, it is known that

$$
V\left(M_{n}(\mathbb{C})\right) \cong \begin{cases}\mathbb{N}_{+} & \text {if } 1 \leq n<\infty \\ \mathbb{N}_{+} \cup\{\infty\} & \text { if } n=\infty\end{cases}
$$

where $\mathbb{N}_{+}=\{0,1, \ldots\}$ (cf. [13, Examples 6.1.4]), and hence

$$
V\left(\sum_{i=1}^{n} \oplus M_{n_{i}}(\mathbb{C})\right) \cong \mathbb{N}_{+}^{\left(k_{1}\right)} \oplus\left(\mathbb{N}_{+} \cup\{\infty\}\right)^{\left(k_{2}\right)},
$$

where $k_{1}$ (resp., $k_{2}$ ) is the number of finite (resp., infinite) $n_{i}$ 's, and for a semigroup $V, V^{(k)}$ denotes the direct sum of $k$ copies of $V$. Our purpose in this section is to prove the following

TheORem 4.1. An operator $T$ on $H$ is the direct sum of irreducible operators if and only if $V\left(W^{*}(T)^{\prime}\right)$ is isomorphic to $\mathbb{N}_{+}^{\left(k_{1}\right)} \oplus\left(\mathbb{N}_{+} \cup\{\infty\}\right)^{\left(k_{2}\right)}$ for some integers $k_{1}$ and $k_{2}, 0 \leq k_{1}, k_{2} \leq \infty$.

Here we briefly recall the definition of $V(\mathcal{A})$. Two projections $p$ and $q$ in $M^{\infty}(\mathcal{A})$, the collection of all finite matrices with entries from $\mathcal{A}$, are said to be equivalent if there is a $v$ in $M^{\infty}(\mathcal{A})$ such that $v^{*} v=p$ and $v v^{*}=q$. The equivalence class containing $p$ is denoted by $[p]$ and the set of all these classes is $V(\mathcal{A}) . V(\mathcal{A})$ is an abelian semigroup with addition defined by

$$
[p]+[q]=[\operatorname{diag}(p, q)],
$$

where $\operatorname{diag}(p, q)$ is the matrix $\left(\begin{array}{ll}p & 0 \\ 0 & q\end{array}\right)$ (cf. [13, Section 6.1]).

Theorem 4.1 will be proved after the following series of lemmas.

Lemma 4.2. Let $P$ and $Q$ be two projections in $W^{*}(T)^{\prime}$ which are orthogonal to each other. If $P$ is unitarily equivalent to $Q$ via a unitary operator in $W^{*}(T)^{\prime}$, then $T \mid(\operatorname{ran} P)$ is unitarily equivalent to $T \mid(\operatorname{ran} Q)$.

Proof. Let $U$ be a unitary operator in $W^{*}(T)^{\prime}$ such that $U P=Q U$, and let $W=U \mid(\operatorname{ran} P)$. Then $W$ is a unitary operator from $\operatorname{ran} P$ onto $\operatorname{ran} Q$ and satisfies $W(T \mid(\operatorname{ran} P))=(T \mid(\operatorname{ran} Q)) W$. 
LEMmA 4.3. Let $T$ be an operator on $H$ with $V\left(W^{*}(T)^{\prime}\right) \cong\left(\mathbb{N}_{+}\right)^{\left(k_{1}\right)} \oplus$ $\left(\mathbb{N}_{+} \cup\{\infty\}\right)^{\left(k_{2}\right)}$, where $0 \leq k_{1}, k_{2} \leq \infty$. Let $l=k_{1}+k_{2},\left\{e_{i}\right\}_{i=1}^{l}$ be the $l$ free generators of $V\left(W^{*}(T)^{\prime}\right)$, and $P \neq 0$ be a projection in $W^{*}(T)^{\prime}$. Then $T \mid(\operatorname{ran} P)$ is irreducible if and only if $[P]=e_{i}$ for some $i$.

Proof. Assume that $T \mid(\operatorname{ran} P)$ is irreducible and let $[P]=\sum_{i=1}^{l} \oplus \alpha_{i} e_{i}$, where the $\alpha_{i}$ 's are integers, $0 \leq \alpha_{i} \leq \infty$. Assume that more than one of the $\alpha_{i}$ 's is nonzero, say, $\alpha_{1}, \alpha_{2} \neq 0$. Then $f \equiv \alpha_{1} e_{1}$ and $g \equiv \sum_{i=2}^{\infty} \oplus \alpha_{i} e_{i}$ are nonzero elements in $V\left(W^{*}(T)^{\prime}\right)$. Hence there exists a natural number $m$ for which there are mutually orthogonal projections $Q$ and $R$ in $M_{m}\left(W^{*}(T)^{\prime}\right)=W^{*}\left(T^{(m)}\right)^{\prime}$ such that $[Q]=f$ and $[R]=g$. If $S=Q+R$, then $[S]=[Q]+[R]=f+g=\sum_{i=1}^{l} \oplus \alpha_{i} e_{i}=[P]$. Hence $S$ and $P \oplus 0^{(m-1)}$ are unitarily equivalent via a unitary operator in $W^{*}\left(T^{(m)}\right)^{\prime}$, where 0 denotes the zero operator on $H$. Lemma 4.2 then implies that $T^{(m)} \mid(\operatorname{ran} S)$ is unitarily equivalent to $T^{(m)} \mid\left(\operatorname{ran}\left(P \oplus 0^{(m-1)}\right)\right)$. But the former equals $\left(T^{(m)} \mid(\operatorname{ran} Q)\right) \oplus\left(T^{(m)} \mid(\operatorname{ran} R)\right)$ while the latter coincides with the irreducible $T \mid(\operatorname{ran} P)$. This is a contradiction. Hence only one of the $e_{i}$ 's can be nonzero, which proves that $[P]=e_{i}$ for some $i$.

Conversely, assume that $[P]=e_{1}$ and $T \mid(\operatorname{ran} P)$ is reducible. Then there are nonzero projections $Q$ and $R$ in $W^{*}(T)^{\prime}$ such that $Q R=0$ and $P=$ $Q+R$. Let $[Q]=\sum_{i=1}^{l} \oplus \alpha_{i} e_{i}$ and $[R]=\sum_{i=1}^{l} \oplus \beta_{i} e_{i}$, where $0 \leq \alpha_{i}, \beta_{i} \leq \infty$ for all $i$. From $e_{1}=[P]=[Q]+[R]=\sum_{i=1}^{l} \oplus\left(\alpha_{i}+\beta_{i}\right) e_{i}$, we deduce that $\alpha_{1}+\beta_{1}=1$ and $\alpha_{i}+\beta_{i}=0$ for all $i \geq 2$. Hence $\alpha_{1}=0$ or $\beta_{1}=0$ and $\alpha_{i}=\beta_{i}=0$ for all $i \geq 2$. This shows that $[Q]=0$ or $[R]=0$, which is a contradiction. Thus $T \mid(\operatorname{ran} P)$ is irreducible.

Lemma 4.4. Assume that $A$ on $H$ is a direct sum of irreducible operators and $B$ on $K$ has no reducing subspace on which it is irreducible. If $X$ is such that $X A=B X$ and $X A^{*}=B^{*} X$, then $X=0$.

Proof. Let $A=\sum_{n=1}^{\infty} \oplus A_{n}$ on $H=\sum_{n=1}^{\infty} \oplus H_{n}$, where $A_{n}$ is irreducible for all $n$. (A similar argument applies if $A$ is the direct sum of finitely many irreducible operators.) Let $X^{*}$ be represented as $\left[X_{1} X_{2} \ldots\right]^{\mathrm{t}}$ from $K$ to $\sum_{n} \oplus H_{n}$. We now show that $X_{1}=0$. Indeed, from $X A=B X$ and $X A^{*}=B^{*} X$ a simple computation yields $X_{1} B=A_{1} X_{1}$ and $X_{1} B^{*}=A_{1}^{*} X_{1}$. Hence $\left(X_{1} X_{1}^{*}\right) A_{1}=A_{1}\left(X_{1} X_{1}^{*}\right)$ and $\left(X_{1} X_{1}^{*}\right) A_{1}^{*}=A_{1}^{*}\left(X_{1} X_{1}^{*}\right)$. Since $A_{1}$ is irreducible, Lemma 3.3 implies that $X_{1} X_{1}^{*}$ is a scalar multiple of identity, say, $X_{1} X_{1}^{*}=\lambda I_{H_{1}}$.

Assuming that $X_{1} \neq 0$, we want to derive a contradiction. Indeed, in this case, we have $\lambda \neq 0$. If $U=\lambda^{-1 / 2} X_{1}$, then $U U^{*}=I_{H_{1}}$ and $Q \equiv U^{*} U$ is a projection on $K$ satisfying $Q B=B Q$. Let $p=I_{H_{1}} \oplus 0$ and $q=0 \oplus Q$ be operators on $H_{1} \oplus K$ and let $p^{\prime}=p \oplus 0$ and $q^{\prime}=q \oplus 0$ on $\left(H_{1} \oplus K\right) \oplus\left(H_{1} \oplus K\right)$. 
Letting $C=A_{1} \oplus B$, we claim that $p^{\prime}$ and $q^{\prime}$ are unitarily equivalent via a unitary operator in $W^{*}\left(C^{(2)}\right)^{\prime}$.

To prove this, let $v=\left(\begin{array}{ll}0 & U \\ 0 & q\end{array}\right)$ on $H_{1} \oplus K$. Then $v$ is a partial isometry in $W^{*}(C)^{\prime}$ with $v v^{*}=p$ and $v^{*} v=q$. Our assertion then follows from [13, Proposition 5.2.12]. By Lemma 4.2, we infer that $C^{(2)} \mid\left(\operatorname{ran} p^{\prime}\right)$ is unitarily equivalent to $C^{(2)} \mid\left(\operatorname{ran} q^{\prime}\right)$. But the former coincides with the irreducible $A_{1}$ and the latter $B \mid(\operatorname{ran} Q)$. Thus $B \mid(\operatorname{ran} Q)$ is irreducible, which contradicts our assumption. This proves that $X_{1}=0$. Similarly, we have $X_{n}=0$ for all $n \geq 2$ and hence $X=0$ as asserted.

We are now ready for the proof of Theorem 4.1.

Proof of Theorem 4.1. The necessity follows from the paragraph before the statement of the theorem. For the sufficiency, we assume that $V\left(W^{*}(T)^{\prime}\right)$ is isomorphic to $\mathbb{N}_{+}^{\left(k_{1}\right)} \oplus\left(\mathbb{N}_{+} \cup\{\infty\}\right)^{\left(k_{2}\right)}$, where $0 \leq k_{1}, k_{2} \leq \infty$. Let $P$ be a projection in some $M_{k}\left(W^{*}(T)^{\prime}\right)=W^{*}\left(T^{(k)}\right)^{\prime}$ ( $k$ is a natural number) such that $[P]$ is one of the free generators of $V\left(W^{*}(T)^{\prime}\right)$. By Lemma 4.3, $T^{(k)} \mid(\operatorname{ran} P)$ is irreducible (here we embed $W^{*}(T)^{\prime}$ into $M_{k}\left(W^{*}(T)^{\prime}\right)$ under the canonical embedding $A \mapsto\left(\begin{array}{cc}A & 0 \\ 0 & 0\end{array}\right)$, which results in the identification of $V\left(W^{*}(T)^{\prime}\right)$ and $V\left(M_{k}\left(W^{*}(T)^{\prime}\right)\right)$; cf. [13, Lemma 6.2.10]). Using Zorn's lemma, we can find a maximal family of mutually orthogonal projections $\left\{P_{j}\right\}_{j=1}^{n}, 1 \leq n \leq \infty$, in $W^{*}\left(T^{(k)}\right)^{\prime}$ such that $T^{(k)} \mid\left(\operatorname{ran} P_{j}\right)$ is irreducible for all $j$. Letting $Q=\sum_{j} P_{j}$, we will show that $Q=I^{(k)}$, the identity operator on $H^{(k)}$.

Assume that this is not the case. Since $Q$ is a projection in $W^{*}\left(T^{(k)}\right)^{\prime}$, the operators $T_{1} \equiv T^{(k)} \mid(\operatorname{ran} Q)$ and $T_{2} \equiv T^{(k)} \mid\left(\operatorname{ran}\left(I^{(k)}-Q\right)\right)$ are acting on nontrivial spaces. Moreover, $T_{1}$ is the direct sum of irreducible operators and $T_{2}$ has no reducing subspace on which it is irreducible. Hence we may apply Lemma 4.4 to infer that $W^{*}\left(T^{(k)}\right)^{\prime}=W^{*}\left(T_{1}\right)^{\prime} \oplus W^{*}\left(T_{2}\right)^{\prime}$. Therefore, $V\left(W^{*}\left(T^{(k)}\right)^{\prime}\right) \cong V\left(W^{*}\left(T_{1}\right)^{\prime}\right) \oplus V\left(W^{*}\left(T_{2}\right)^{\prime}\right)$ (cf. [13, Proposition 6.2.1]). Since both $V\left(W^{*}\left(T^{(k)}\right)^{\prime}\right)=V\left(W^{*}(T)^{\prime}\right)$ and $V\left(W^{*}\left(T_{1}\right)^{\prime}\right)$ are torsion-free semigroups, the same is true for $V\left(W^{*}\left(T_{2}\right)^{\prime}\right)$. Let $R$ be a projection in $W^{*}\left(T_{2}^{(m)}\right)$ ( $m$ is a natural number) for which $[R]$ is one of the free generators of $V\left(W^{*}\left(T_{2}\right)^{\prime}\right)$. From Lemma 4.3, we know that $T_{2}^{(m)} \mid(\operatorname{ran} R)$ is irreducible. Arguing as above, we can find a nonzero projection $Q_{1}$ in $W^{*}\left(T_{2}^{(m)}\right)^{\prime}$ such that $T_{3} \equiv T_{2}^{(m)} \mid\left(\operatorname{ran} Q_{1}\right)$ is the direct sum of irreducible operators and $T_{4} \equiv T_{2}^{(m)} \mid\left(\operatorname{ran}\left(I-Q_{1}\right)\right)$ has no reducing subspace on which it is irreducible.

Applying Lemma 4.4, we find that $W^{*}\left(T_{2}^{(m)}\right)^{\prime}=W^{*}\left(T_{3}\right)^{\prime} \oplus W^{*}\left(T_{4}\right)^{\prime}$. Thus $Q_{1}$ commutes with every operator in $W^{*}\left(T_{2}^{(m)}\right)^{\prime}$, that is, $Q_{1}$ is in $W^{*}\left(T_{2}{ }^{(m)}\right)^{\prime \prime}=W^{*}\left(T_{2}^{(m)}\right)$ by the von Neumann double commutant theorem. 
Therefore, $Q_{1}$ is of the form $S^{(m)}$, where $S$ is a nonzero projection in $W^{*}\left(T_{2}\right)$, and hence $T_{3}=T_{2}^{(m)} \mid\left(\operatorname{ran} Q_{1}\right)=\left(T_{2} \mid(\operatorname{ran} S)\right)^{(m)}$. Since $T_{3}$ is the direct sum of irreducible operators, the same is true for $T_{2} \mid(\operatorname{ran} S)$ by Proposition 3.6. This contradicts the fact that $T_{2}$ has no reducing subspace on which it is irreducible. Hence we must have $Q=I^{(k)}$. Thus $T^{(k)}$ is a direct sum of irreducible operators. By Proposition 3.6, the same is true for $T$.

We end this paper by noting that Theorem 4.1 cannot be generalized to arbitrary $C^{*}$-algebras, that is, a (unital) $C^{*}$-algebra $\mathcal{A}$ with $V(\mathcal{A})$ isomorphic to $\mathbb{N}_{+}^{\left(k_{1}\right)} \oplus\left(\mathbb{N}_{+} \cup\{\infty\}\right)^{\left(k_{2}\right)}, 0 \leq k_{1}, k_{2} \leq \infty$, may not be $*$-isomorphic to $\sum_{i} \oplus M_{n_{i}}(\mathbb{C})$, where $1 \leq n_{i} \leq \infty$. An example of such a $C^{*}$-algebra is $\mathcal{A}=\{\lambda I+K: \lambda \in \mathbb{C}, K$ a compact operator on $H\}$, where $H$ is an infinitedimensional separable Hilbert space. It can be verified that $V(\mathcal{A})$ is isomorphic to $\mathbb{N}_{+} \cup\{\infty\}$ (cf. [13, Examples 6.1.4]), but $\mathcal{A}$ is not $*$-isomorphic to $\mathcal{B}(H)$ since their $K_{0}$-groups are different (cf. [13, Examples 6.2.3]). Whether there are examples of such von Neumann algebras seems to be unknown.

Acknowledgements. The research of the first two authors was supported by the National Natural Science Foundation of China and the Mathematical Center of the State Education Commission of China, while that of the third author by the National Science Council of the Republic of China (Taiwan). The third author would like to thank Peter Rosenthal for providing an example which clarified his misconception on commuting projections.

\section{References}

[1] E. A. Azoff, C. K. Fong and F. Gilfeather, A reduction theory for non-self-adjoint operator algebras, Trans. Amer. Math. Soc. 224 (1976), 351-366.

[2] J. B. Conway and T. A. Gillespie, Is a self-adjoint operator determined by its invariant subspace lattice?, J. Funct. Anal. 64 (1985), 178-189.

[3] M. J. Cowen and R. G. Douglas, Complex geometry and operator theory, Acta Math. 141 (1978), 187-261.

[4] P. R. Halmos, Irreducible operators, Michigan Math. J. 15 (1968), 215-233.

[5] —, Two subspaces, Trans. Amer. Math. Soc. 144 (1969), 381-389.

[6] D. A. Herrero, Approximation of Hilbert Space Operators, Vol. I, Pitman, Boston, 1982.

[7] C. Jiang and Z. Wang, Strongly Irreducible Operators on Hilbert Space, Longman, Harlow, 1998.

[8] R. V. Kadison and I. M. Singer, Three test problems in operator theory, Pacific J. Math. 7 (1957), 1101-1106.

[9] S.-C. Ong, What kind of operators have few invariant subspaces?, Linear Algebra Appl. 95 (1987), 181-185.

[10] H. Radjavi and P. Rosenthal, Invariant Subspaces, Springer, New York, 1973.

[11] M. Takesaki, Theory of Operator Algebras I, Springer, New York, 1979. 
[12] D. Voiculescu, A non-commutative Weyl-von Neumann theorem, Rev. Roumaine Math. Pures Appl. 21 (1976), 97-113.

[13] N. E. Wegge-Olsen, K-theory and $C^{*}$-algebras, Oxford Univ. Press, Oxford, 1993.

Department of Mathematics

Hebei University of Technology

Tianjin 300130, China

E-mail: fangjs@263.net

Department of Applied Mathematics

National Chiao Tung University

Hsinchu 300, Taiwan

E-mail: pywu@math.nctu.edu.tw
Department of Mathematics Hebei Nomal University Shijiazhuang 050016, China E-mail: cljiang@hebtu.edu.cn

Received September 21, 2000

Revised version July 31, 2002 